\title{
Primary submicron particles from early stage asphaltene precipitation revealed in situ by total internal reflection fluorescence microscopy in a model oil system
}

\author{
Jia Meng ${ }^{\mathrm{a}}$, Jae Bem You ${ }^{\mathrm{a}, \mathrm{c}}$, Hao Hao ${ }^{\mathrm{b}}$, Xiaoli Tan ${ }^{\mathrm{a}, *}$, Xuehua Zhang ${ }^{\mathrm{a}, *}$ \\ ${ }^{a}$ Department of Chemical and Materials Engineering, University of Alberta, Alberta T6G 1H9, Canada \\ ${ }^{\mathrm{b}}$ Central Faculty Office (FSET), Swinburne University, Melbourne 3122, Australia \\ ${ }^{\mathrm{c}}$ Physics of Fluids Group, Max Planck Center Twente for Complex Fluid Dynamics, JM Burger Center for Fluid Dynamics, Mesa+, Department of Science and Technology, \\ University of Twente, 7500 AE Enschede, The Netherlands
}

\section{A R T I C L E I N F O}

\section{Keywords:}

Asphaltene precipitation

Microchamber

In-situ observation

Primary submicron particles

\begin{abstract}
A B S T R A C T
Paraffinic froth treatment (PFT) is an essential step in oilsands extraction. The ability to quantitatively understand and control asphaltene precipitation induced by solvent dilution is key to technology innovation in PFT process. In this work, we investigate the early stage of asphaltene precipitation in a model oil system in response to diffusive solvent addition in quasi-2D confinement. Using total internal reflection fluorescence microscope, we provide direct visualization of the size distribution and structural characteristics of asphaltene precipitates with a spatial resolution of $\sim 200 \mathrm{~nm}$ and temporal resolution of $250 \mathrm{~ms}$. Our results show the correlation between the size and number of the asphaltene particles and the concentration of the paraffinic solvent in the diluent. Notably the aggregates were found to consist of primary submicron particles with a similar size from $200 \mathrm{~nm}$ to $400 \mathrm{~nm}$ in radius. These particles may be the primary elementary units that aggregate and form bigger particles via aggregation. The growth time of asphaltene particles decreases with increase n-pentane concentration in the observation area. The findings from this work provide new insight into the effects of solvent mixing on the size distribution and morphological characteristics of asphaltene precipitates that are important for association with water and solids and separation properties.
\end{abstract}

\section{Introduction}

Asphaltene is classified as the most complex and heaviest component in heavy oil, consisting of polynuclear aromatic species with different molecular weight, polarity, and heteroatom content. As a solubility class, asphaltene is insoluble in paraffinic solvents and soluble in aromatic solvents [1]. In the industrial process of oilsands extraction, bitumen with a considerable amount of fine solids and water is separated from oilsands ore by warm-water extraction to form bitumen froth. The froth typically consists of $60 w t \%$ bitumen, $10 w t \%$ mineral solids, and $30 w t \%$ water [2]. In paraffinic froth treatment (PFT), solids and water are removed from the bitumen froth by adding paraffinic solvents at the solvent/bitumen ratio (S/B ratio) above a critical value, which triggers asphaltene precipitation and agglomeration with water drops and solids. Controlled asphaltene precipitation in PFT process is a key parameter to yield bitumen product in both high quality and high recovery $[2,3]$.

For several decades, extensive research has been conducted to understand molecular or colloidal structures, solubility and phase behavior of asphaltene in various media including solvents and bitumen. However, dilution-induced asphaltene precipitation in bitumen froth treatment is still one of the most important and challenging topics. In addition to complexity in chemical and physical properties of asphaltene and bitumen, asphaltene precipitation induced by solvent dilution involves dynamical processes over multiple length scales (from nanometer to millimeter) and varied time scale (from seconds to days). It is known that the onset and quantity of asphaltene precipitation are related to the solvent type, solvent/bitumen (S/B) ratio, temperature and pressure. For a given solvent, asphaltene precipitation occurs over a range of $\mathrm{S} / \mathrm{B}$ ratios [1]. The size, density and structure of the asphaltene precipitates are important for the separation properties of their aggregates with water droplets and solids.

\footnotetext{
* Corresponding authors.

E-mail addresses: xiaolit@ualberta.ca (X. Tan), xuehua.zhang@ualberta.ca (X. Zhang).
} 
However, the morphology and structure of the precipitates are not well defined. The settling behavior suggests that asphaltene precipitates are porous and tenuous fractal structures with fluid trapped inside. A quicker settling rate represents a larger fractal dimension and more compact particles [4]. The settling rate depends on the type of solvent precipitant. For example, the rate of n-pentane precipitated asphaltene is two magnitudes higher than that of $\mathrm{n}$-heptane precipitated asphaltene [4]. For a given type of precipitant, settling rate increases with the concentration of precipitant and then decreases at approximately $75 \%$ [4]. Based on the mean diameter of the precipitates, two classical models are applied to approximately describe the growth dynamics: diffusionlimited aggregation (DLA) and reaction-limited aggregation (RLA) $[5,6]$. Transition from one model to the other was observed at different $\mathrm{S} / \mathrm{B}$ ratios, although the mechanism for such transition is not fully understood [5-7].

In parallel, liquid-liquid microphase separation induced by solvent dilution is ubiquitous in many other technological and industrial processes. A simple example is known as 'Ouzo' effect in a ternary mixture of oil, ethanol and water [8]: When water is added into a clear Greek alcoholic drink Ouzo, anise oils in the drink become oversaturated by dilution and spontaneously form nanodroplets in the cloudy mixture [9]. The same effect can also be seen when eucalyptus disinfectants and mosquito repellents in alcohol solutions are diluted with water [10]. In liquid-liquid microextraction, oil microdroplets from the spontaneous phase separation by dilution are the basic unit to concentrate and separate trace hydrophobic analytes from aqueous samples [11]. Gas molecules, small hydrophobic organics, lipids or polymers dissolved in a polar organic solvent all exhibit similar effects, forming bubbles, or nanoparticles upon dilution by a poor solvent. The process is also referred to as solvent exchange [12], nanoprecipitation [13], solvent displacement or solvent shifting $[14,15]$.

The key feature of the above spontaneous liquid-liquid phase separation is that the growth dynamics of individual domains is determined not only by concentration of the compositions (thermodynamic aspects), but also by the temporal and spatial characteristics of the mixing process of the solvents (dynamic aspects) [8]. How the solvent is added has a great impact on the size distribution, morphological characteristics, chemical and physical properties of the formed new phase. The dilutioninduced microphase formation is complex, influenced by several factors including solution composition [16] and mixing of the solvent and the solution [8]. Latest advance made in well-defined systems has provided a solid foundation to understanding another dilution-induced phase separation process in heavy oils: asphaltene precipitation. The experimental techniques and theoretical frameworks are transferable to the study of early stage dynamics of asphaltene precipitation induced by mixing with a paraffinic solvent.

There are knowledge gaps in early stage dynamics of asphaltene precipitation induced by paraffinic solvent addition. Direct visualization of the size distribution and structural characteristics of asphaltene precipitates from an early stage is challenging, largely due to the high optical density of the mixture mainly from asphaltene fractions. Measurements with improved spatial resolution were recently performed by using confocal laser-scanning microscopy in a transmission mode [7]. Size distribution of asphaltene aggregates in various solvents and precipitants were observed as a function of time when a very thin film of the mixture was confined between two plates [7]. The amount and the size distribution of asphaltene precipitates after phase separation were also studied by a confocal microscope and image processing technique $[17,18]$. In the optical measurements above-mentioned, the concentration of asphaltene in the crude oil/solvent solution must remain very low $(<0.2 \mathrm{mg} / \mathrm{ml})$ to allow enough intensity of the light to pass through the samples. Therefore the measurements were either far below the concentration regimes in bitumen, or missed the entire early stage dynamics of the precipitation, due to the ratio of solvent already high above the critical ratio.

Total internal reflection fluorescence microscopic system (TIRF) is a technique that can largely overcome experimental difficulties from asphaltene while providing high temporal and spatial resolutions. In the configuration of TIRF, the light travels within a substrate (usually a thin glass slide) through total internal reflection and creates an evanescent field above the substrate [19]. The selective excitation and visualization of species in TIRF is confined in a narrow zone of the evanescent field generated from the total internal reflection. The sampling zone in TIRF is approximately $150 \mathrm{~nm}$ adjacent to a substrate as well as what on the surface. The probe depth into the sampling zone depends on the refractive index of the media. The strong scattering background from the medium outside of the substrate can be largely neglected, which is the most desirable feature of TIRF configuration, compared to a transmission or reflection mode of an optical microscope. The difficulty from the high optical density will be largely overcome, due to the thin zone of the evanescent field. TIRF allows for following the microphase separation during solvent mixing.

The aim of this work is to in situ study dynamics of asphaltene precipitation in response to different mixing processes of a model paraffinic solvent (i.e. n-pentane). Employing the experimental techniques of TIRF microscope for mixing in a quasi-2D chamber, our measurements will reveal the onset and temporal evolution of asphaltene precipitates during the dilution. The measurements will provide high temporal $(\sim$ $0.25 \mathrm{~s}$ ) and spatial $(\sim 200 \mathrm{~nm})$ resolutions at high asphaltene concentration. This work will reveal early stage asphaltene precipitation during diffusive mixing with a paraffinic solvent. Although convective mixing takes place in many solvent-induced precipitation processes including PFT, locally diffusive mixing may happen, particularly for a large volume and/or viscous liquids. Furthermore, diffusive mixing process allows us to understand the roles of solvent diffusion in the precipitation on a microscopic scale, laying the basis for a quantitative understanding of asphaltene precipitation under more complicated mixing conditions. Our results show that the sizes of asphaltene precipitates are associated with mixing conditions.

From TIRF images, we quantify the size distribution, particle quantity and surface coverage of asphaltene precipitates. Size of precipitate particles is an important parameter for asphaltene precipitation modeling $[20,21]$, associated with the degree of flocculation of the primary submicron particles. As the sedimentation of asphaltene precipitates is essential for sweeping down water and solids from bitumen in PFT [2,22,23], our work implies that mixing conditions may be an approach to form large aggregates of asphaltene with extended structures for efficiently cleaning up water and solids.

\section{Experiment}

\subsection{Chemicals and sample preparation}

Asphaltene used in this study was n-pentane precipitated asphaltene supplied by Quadrise Canada Corporation, and it was used as received. Toluene (ACS grade, Fisher Scientific, 99.9+\%) was used as the solvent for asphaltene. The diluent was n-pentane (Fisher Scientific, 98\%). Nile Red (Fisher Scientific) was used for microchamber characterization. CHNS/O Analyzer (Flash 2000, Thermo Scientific) was used to measure the organic element composition of asphaltene in the asphaltene sample, and the composition is $85.3 \pm 0.9 w t . \%$ of carbon, $8.2 \pm 0.1 w t \%$ of hydrogen, $3.7 \pm 0.1 w t . \%$ of sulfur and $1.0 \pm 0.0 w t . \%$ of nitrogen.

The asphaltene in toluene solution was prepared as following: $20 \mathrm{~g}$ asphaltene was first dissolved in $200 \mathrm{~mL}$ toluene and then the mixture was shaken for $30 \mathrm{~min}$ and left still at room temperature for 1 day till asphaltene was fully dissolved. The mixture was filtered by using a PVFD filter paper with $0.22 \mu \mathrm{m}$ pore size (Millipore) to remove any inorganic solids $>0.22 \mu \mathrm{m}$ in the asphaltene sample. After removal of toluene by rotatory evaporator, the obtained asphaltene is treated as both inorganic solids and solvent free asphaltene. It is worth noting that trace amount of inorganic solids with $<0.22 \mu \mathrm{m}$ size may still remain in the asphaltene after the filtration, but their impact on asphaltene precipitation was 
negligible in this study. $1.7 \mathrm{~g}$ of the treated asphaltene sample was redissolved in toluene with a total volume of $100 \mathrm{~mL}$. The mixture was well shaken for $30 \mathrm{~min}$ and left for 1 day for asphaltene to be fully dissolved in toluene. The as-prepared asphaltene in toluene solution is named as 'solution A' with a concentration of $17 \mathrm{~g} / \mathrm{L}$.

The solvent (named 'solution B') used to precipitate asphaltene in all experiments was a mixture of n-pentane and toluene with the initial concentration of n-pentane in solution B $\left(C_{B}^{\text {pen }}\right)$ from $70 \%$ to $100 \%$, as shown in Table 1.

\subsection{In-situ detection of asphaltene precipitates using quasi-2D microfluidic device and TIRF}

A quasi-2D microfluidic device consisting of a shallow main channel (height $=20 \mu \mathrm{m}$ ) and a deep side channel (height $=260 \mu \mathrm{m}$ ) was used for the in situ detection of asphaltene precipitation. Diffusive mixing between toluene and n-pentane was confirmed through fluorescence microscopy by doping toluene with Nile red. A minimum distance from the side channel to the main channel required to achieve fully diffusive mixing was obtained from the fluorescence characterization.

For the precipitation of asphaltene, the quasi-2D microfluidic device was pre-filled with a solution A. Solution B was introduced through the side channel at $5 \mathrm{~mL} / \mathrm{h}$ continuously replacing solution A. The process proceeds till all solution A displaced from the chamber as sketched in Fig. 1a. Once in the side channel, solution B diffused through the main channel to mix with solution A. No apparent convective flow was observed due to the shallow height of the main channel. Detailed characterization of diffusive mixing in the quasi-2D microfluidic device was described elsewhere [24,25].

As solution B mixed with solution A, precipitation of asphaltene was observed using TIRF equipped with $60 \times / 1.49$ NA objective (DeltaVision OMX Super-resolution microscope, GE Healthcare UK limited, UK). The fluorophores in the asphaltene solution were excited with a green laser with wavelength $\lambda=488 \mathrm{~nm}$ and fluorescence emission was detected at $576 \mathrm{~nm}$. The images were collected in a field of view of 82.5 $\mu m \times 82.5 \mu m$ distanced more than $80 \mu m$ from the side channel to eliminate the edge effect. The refractive indices of asphaltene (RI = $1.72)$, toluene $(R I=1.50)$, and n-pentane $(R I=1.36)$ needed for TIRF were taken from literature [26-28]. We note that the RI of 1.72 from the literature is not a universal value, possibly varying with the source of asphaltene. However, the contrast in TIRF imaging only requires the difference between asphaltene and the surrounding medium. As asphaltene precipitated out, fluorescence signal was quenched and the precipitates appeared dark. Although TIRF can detect objects on the solid surface as well as in the evanescent field, the precipitates in the images at our final stage are all on the surfaces, as our solution is already $\mathrm{n}$-pentane without precipitates. Even if some objects are in the bulk, they may not be visible to our TIRF images if they move around. So even our images showing the dynamical process, the precipitates are mainly on the surface.

Confocal Laser Scanning Microscopy was performed on a Leica SP8 STED (Stimulated Emission Depletion) Falcon system (Leica microsystem, Germany) based on an inverted microscope (Leica DM8). The dried samples in ambient temperature were imaged with a $100 \times / 1.4$ NA immersion objective using $470 \mathrm{~nm}$ laser excitation in order to validate the image collected from TIRF and morphology of precipitated

Table 1

Composition of solvents. For all cases, solution A is $17 \mathrm{~g} / \mathrm{L}$ of asphaltene in toluene. $C_{B}^{\text {pen }}$ is the initial concentration of $n$-pentane in solution $B$

\begin{tabular}{ccc}
\hline$C_{B}^{\text {pen }}($ vol.\%) & n-Pentane (vol.\%) & Toluene (vol.\%) \\
\hline 70 & 70 & 30 \\
80 & 80 & 20 \\
90 & 90 & 10 \\
100 & 100 & 0 \\
\hline
\end{tabular}

asphaltene processed from the image analysis. The same samples after TIRF experiment were used for the purpose. The solvent (i.e. n-pentane), which is already free of asphaltene after TIRF experiment, was removed by evaporation overnight, while asphaltene particles remain on the substrate. The fluorescence emission signal was collected between 478 $\mathrm{nm}$ to $670 \mathrm{~nm}$ range at a pixel size of $105 \mathrm{~nm}$ and using a dwelling time of $1.44 \mu s(400 \mathrm{~Hz})$. Typically, 4 time line averaging was used to collect the images. The spatial resolution of confocal microscope is $120 \mathrm{~nm}$. If the spacing between two neighboring domains is smaller than the spatial resolution, the separation between the domains cannot be resolved in the images. A more thorough description of the system could be found in the literature [29].

\subsection{Image analysis of asphaltene precipitates}

The series images extracted from the video frames were processed and analysed using a software programmed in MATLAB. Microscopic images often suffered the problems, such as low contrast, noise and uneven background. Several image processing techniques were used to enhance the image, including stretching pixel intensity range, median filter, Gaussian noise filter and top-hat filtering. The enhanced image was then binarized with a selected threshold $(T)$. If the intensity value of the pixel is smaller than $T$, it was converted to 0 . The choice of $T$ was arbitrary to a certain extent with a relationship with the maximum intensity in the whole image $\left(I_{\max }\right)$. The threshold was varied from $0.5 I_{\max }$ to $0.8 I_{\max }$ to capture as many particles as possible. The arbitrary of choosing threshold leads to approximately $30 \%$ uncertainty of the data based on tests. The objects smaller than $200 \mathrm{~nm}$ were removed using area opening and the objects along the image border were masked out because they are likely noise of the image as the size is already close to the spatial resolution of TIRF. The objects larger than $200 \mathrm{~nm}$ in radius (i.e. $400 \mathrm{~nm}$ in diameter) were reliable. For the $80 \mathrm{~nm}$ pixel size TIRF camera, objects larger than $200 \mathrm{~nm}$ radius contain more than 20 pixels.

The software measured the set of properties for each object in the binary image, including object centroid, area size, perimeter and radius of the precipitates. The centroid provided location information for further tracking the objects in the series images collected at different time. The white dots with irregular shape corresponded asphaltene particles, as shown in Fig. 1c. The dots were then outlined by blue lines and the equivalent radius of particles was defined as the radius of the circle that has the same area as the irregular shape, as shown in Fig. 1d and 1 e. Particle quantity was obtained by counting the number of the white dots divided by the total observation area. Surface coverage was calculated by summing the areas occupied by particles divided by the total observation area.

\section{Results}

\subsection{Morphology of early stage precipitates: primary submicron particle sizes}

High spatial resolution images enabled by TIRF allow us to resolve the asphaltene aggregates formed by several particles. The asphaltene particles are dispersed and there is little evidence of overlap when the images were taken. As shown in Fig. 2, in the primary submicron particle study, aggregates are isolated into primary submicron particles. We define the primary submicron particles are the population with the most probable distribution. These primary submicron particles are larger than the spatial resolution limit (i.e. $200 \mathrm{~nm}$ ), and therefore the images may reliably reveal their sizes. The aggregates are referred to as fractal aggregates in the confocal study.

The asphaltene particle parameters at final state were obtained by averaged value of three rounds of experiments after injecting solution $B$ into the microfluidic device for sufficient time. Final state is defined as the state at which the asphaltene particle parameters (i.e. mean radius, particle quantity and surface coverage) are stable and the asphaltene in 
(a)

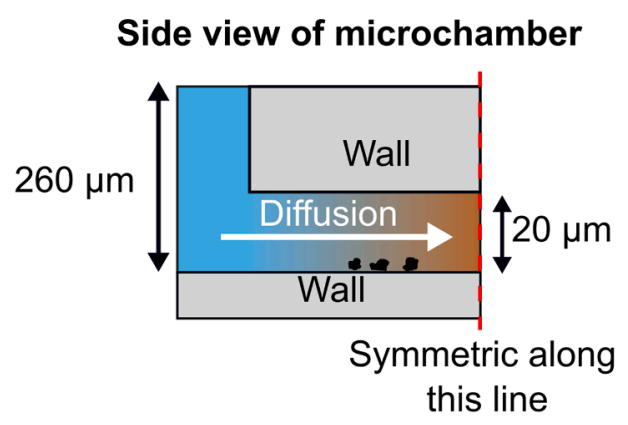

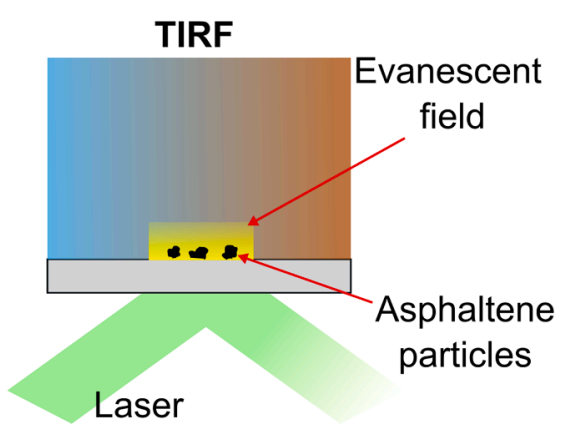

Fig. 1. a) Schematic representation of the microchamber and TIRF setup. b) Representative TIRF image of asphaltene particles when asphaltene solution is displaced by solution B (n-pentane solution). The asphaltene particles appear as black dots. c) Binary image processed by MATLAB code based on TIRF image with white dots representing asphaltene particles. d) Outline asphaltene particles for further analysis. Scale bar is $10 \mu \mathrm{m}$. e) Zoomed-in image of an outlined particle to show the irregular structure of asphaltene particles. Scale bar is $1 \mu \mathrm{m}$.
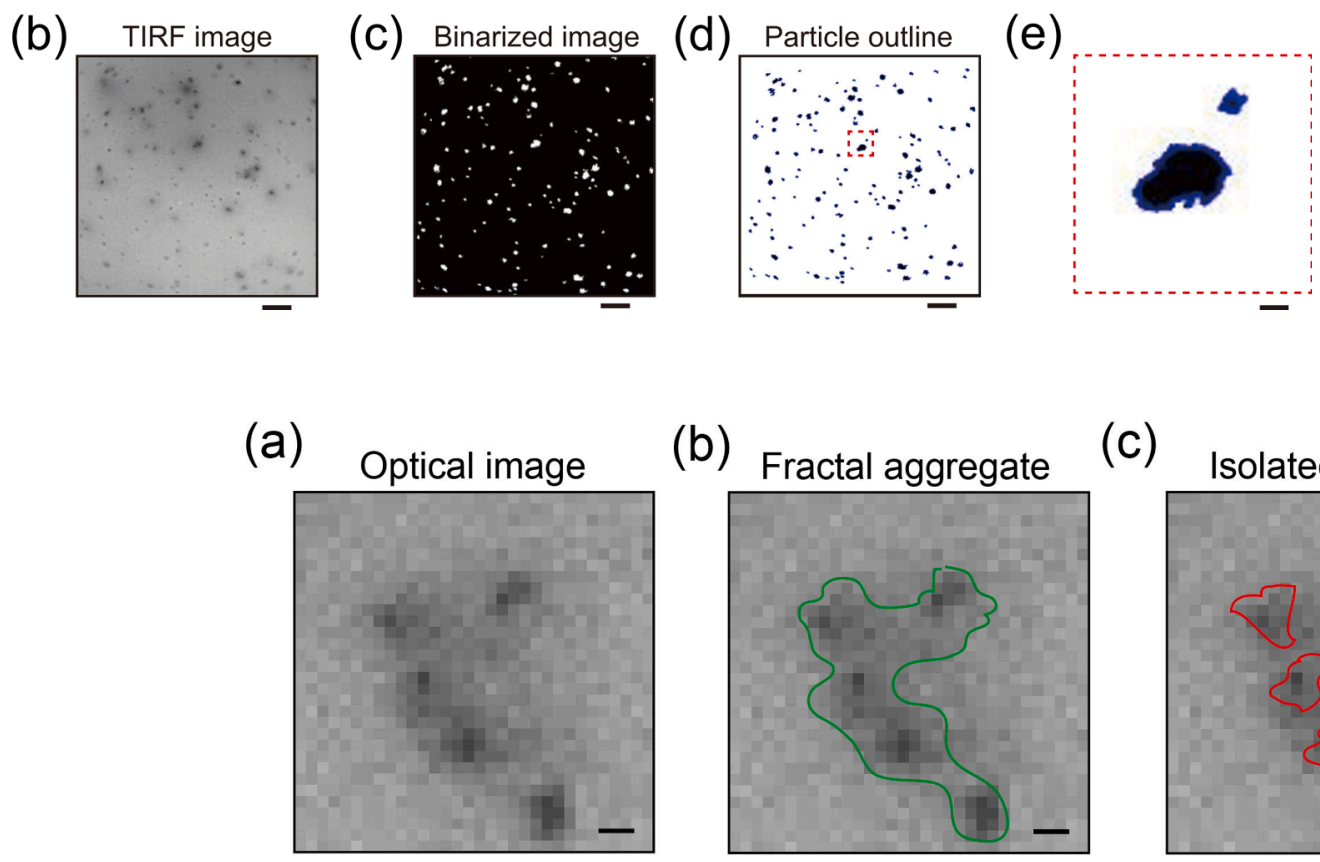

(b)



(c)



Fig. 2. a) Snapshot of an asphaltene aggregate at $C_{B}^{\text {pen }}$ of $90 \%$. b) Fractal structure of the asphaltene aggregate. c) Isolate aggregate to primary submicron particles. Scale bar is $1 \mu \mathrm{m}$.

the mixture does not lead to further precipitation in measured time duration.

Fig. 3a shows images of the final state of precipitated asphaltene particles over a large field of view for variety of particle sizes at different $\mathrm{n}$-pentane concentration in solution B. The zoomed-in images correspond to the asphaltene particles in the corresponding color square in the large field of view. The detailed structure of asphaltene particles are revealed in the images. The asphaltene particles are either primary submicron particles or aggregates formed by several primary submicron particles.

To study the primary submicron particle size, the aggregates were isolated into primary submicron particles. The primary submicron particle size was measured by counting pixels. Fig. 3b shows the particle equivalent radius at $C_{B}^{\text {pen }}$ of $70 \%$ to $100 \%$. The data were collected based on a $82.5 \mu \mathrm{m} \times 82.5 \mu \mathrm{m}$ area in each group of n-pentane concentration. Remarkably, the majority of primary submicron particle radii are distributed in a narrow range of $200 \mathrm{~nm}-400 \mathrm{~nm}$, independent of $C_{B}^{\text {pen }}$. The average radius is around $300 \mathrm{~nm}$.

The primary submicron particle size of precipitated asphaltene was further confirmed by confocal microscope, as shown in Fig. 4, where the confocal images were captured in air after precipitation with $100 \% \mathrm{n}$ pentane to avoid threshold influences on the detection in the solution. With neglecting the data of aggregates, the averaged equivalent particle radius measured from confocal images on the precipitates is around 250 $\mathrm{nm}$, which is comparable to the value obtained from TIRF experiment in solutions. The average size of the primary submicron particle in the solvent is slightly larger than that in the dry state, consistent with swelling behavior of asphaltene in the solvent [30]. The results verified the presence of primary submicron particles, and the reliability of using TIRF to monitor the asphaltene precipitation solutions. Fig. $4 \mathrm{~b}$ has a peak from $0.2 \mu \mathrm{m}$ to $0.4 \mu \mathrm{m}$ in the relative frequency distribution based on quantity of asphaltene particles, representing primary submicron particles whose radius is from $0.2 \mu \mathrm{m}$ to $0.4 \mu \mathrm{m}$. There are also some particles whose equivalent radius distribute from $0.4 \mu \mathrm{m}$ to a few microns, representing aggregates with different sizes coexist with primary submicron particles. The absence of particles smaller than 0.2 micron is the result of the detection limit. Noise and small particles cannot be distinguished beyond the detection limit.

\subsection{Influence of initial solvent concentration on asphaltene precipitates}

Three characteristics of the asphaltene particles are studied in this section, including particle size distribution, particle quantity and surface coverage of asphaltene particles. Although asphaltene aggregates are formed by several primary submicron particles, aggregates were treated as single units rather than counting primary submicron particles in it 
(a)
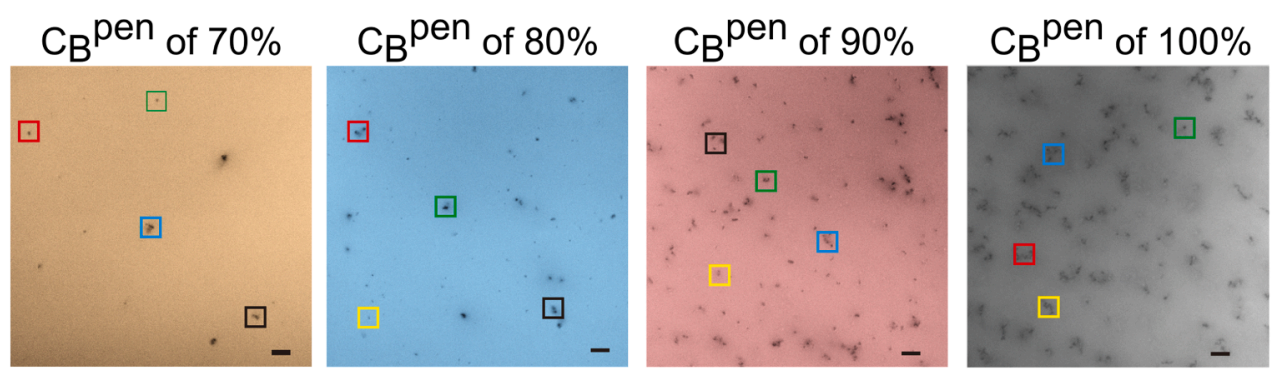

(b)
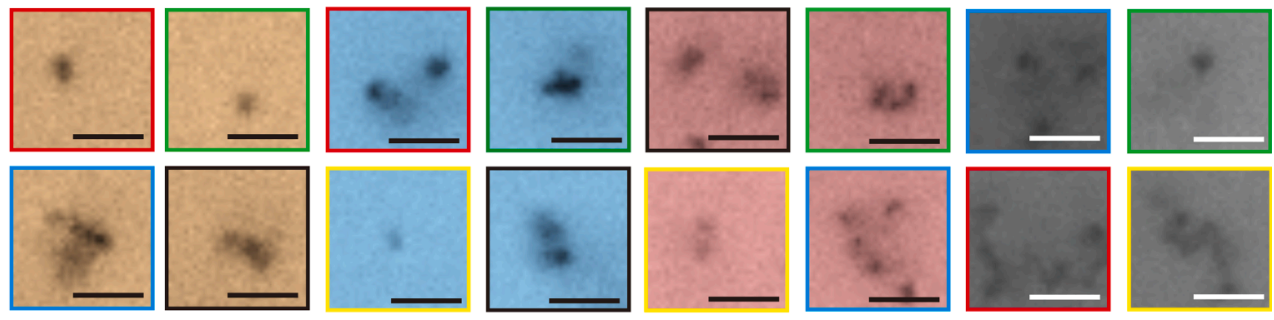

Fig. 3. a) TIRF images of asphaltene particles at the concentration of $n$ pentane in solution B $\left(C_{B}^{\text {pen }}\right)$ of $70 \%$ to $100 \%$. Scale bar is $5 \mu \mathrm{m}$. b) Zoomed-in view of primary submicron particles shown in the TIRF images at locations with respective color box. Note: the images have been false-colored. Scale bar is $5 \mu \mathrm{m}$. c) Primary submicron particle size at different n-pentane concentration in solution B. Error bar shows the standard deviation of the primary submicron particle radius based on three rounds of experiments.

(c)

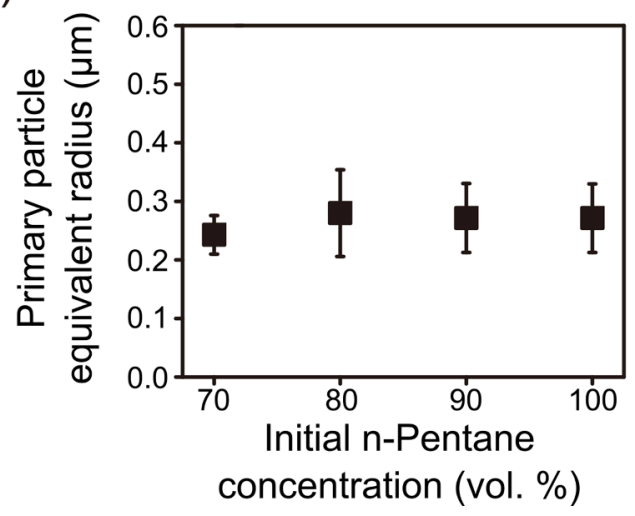

(a)

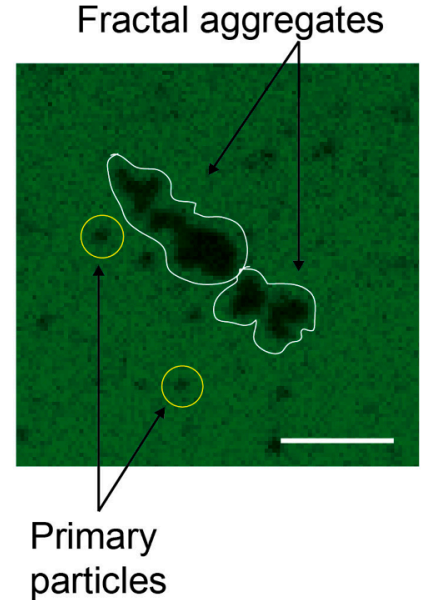

(b)

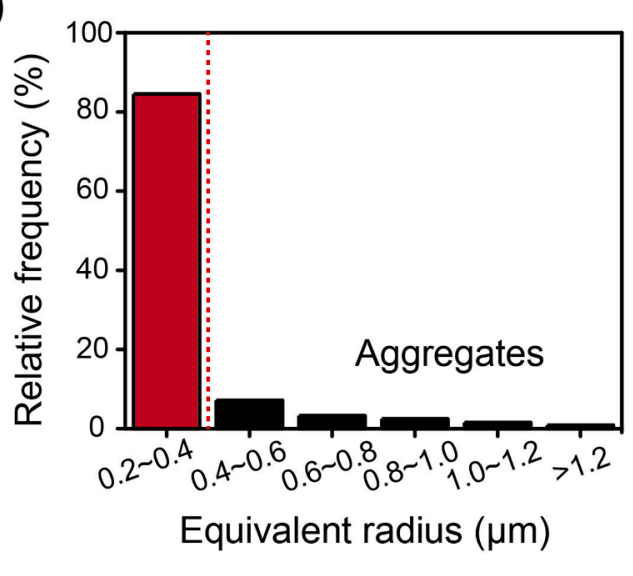

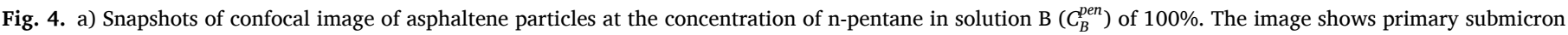

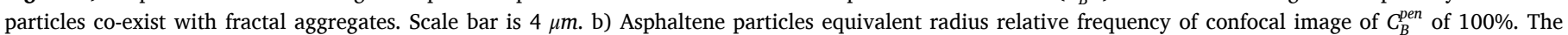
column in red corresponds to the primary submicron particle in the image of a).

when measuring particle size and counting particle quantity. Particle quantity and surface coverage were normalized by the unit area in the field of view.

The morphology of asphaltene at final state is influenced by $C_{B}^{\text {pen }}$.
Fig. 5a shows particle quantity increases with n-pentane concentration in solution B. The asphaltene particle quantity at $C_{B}^{\text {pen }}$ of $70 \%$ is around 1 \pm 1 per $1000 \mu \mathrm{m}^{2}$, and is around $12 \pm 5$ per $1000 \mu \mathrm{m}^{2}$ at $C_{B}^{\text {pen }}$ of $100 \%$. Fig. $5 \mathrm{~b}$ shows asphaltene surface coverage increases with n-pentane 
(a)

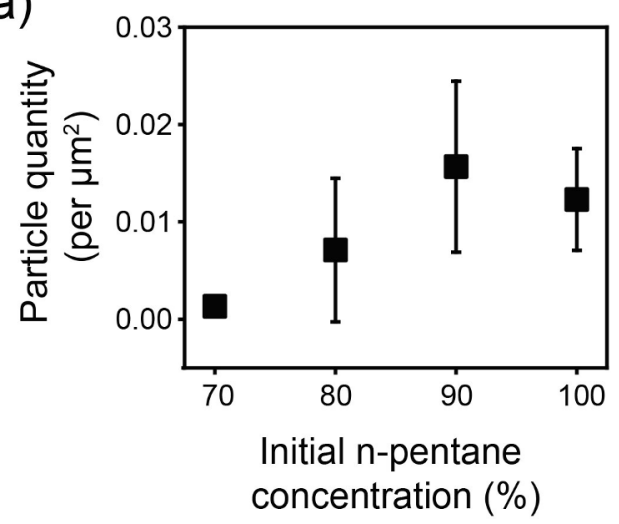

(b)

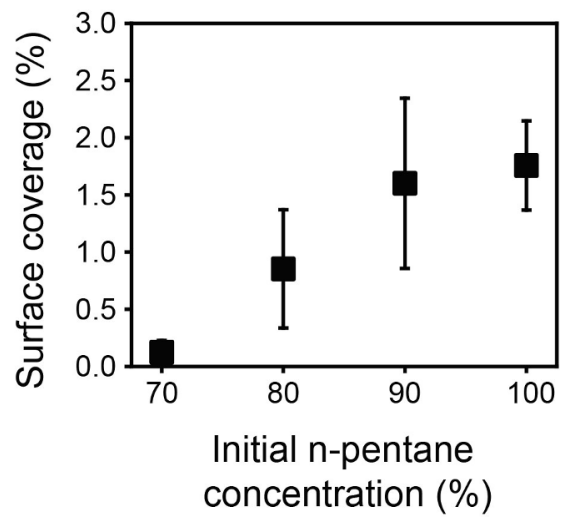

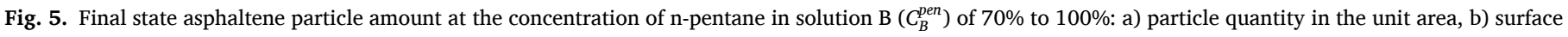
coverage of asphaltene particles.

concentration in solution B. The surface coverage at $C_{B}^{\text {pen }}$ of $70 \%$ is around $0.12 \pm 0.10 \%$, while the surface coverage reaches around $1.76 \pm$ $0.39 \%$ at $C_{B}^{\text {pen }}$ of $100 \%$. For precipitates that are too small to settle on the surface the precipitates can come close to the surface through Brownian motion, and stay there through non-specific interactions with the surface. Surface quantity and coverage are approximately proportional to the concentration of asphaltene precipitates in the bulk mixture. The concentration of precipitates in our experiments may not be constant with time. The surface coverage represents the concentration of precipitates in the entire process of solvent mixing.

Fig. 6 shows influence of $C_{B}^{\text {pen }}$ on asphaltene particle size distribution at final state. Experiments were repeated three times for each condition in order to reduce errors. There are always two types of particles, regardless of $C_{B}^{\text {pen }}$ : primary submicron particles and aggregates. At the final state, the size distribution of the asphaltene has an obvious peak of primary submicron particles, as shown in Fig. 6. The corresponding primary submicron particle size is consistent with the confocal result in Fig. 4. The height of the peak appears to be lower than that in the confocal results. The difference may be mainly caused by the state of samples, where confocal microscope captures all the primary submicron particles in dried state while some of these particles in TIRF experiment are mobilized as a suspension in the solution medium. Asphaltene aggregates with different sizes are formed. TIRF results also show the existence of aggregates with different sizes from $0.4 \mu \mathrm{m}$ to a few microns,

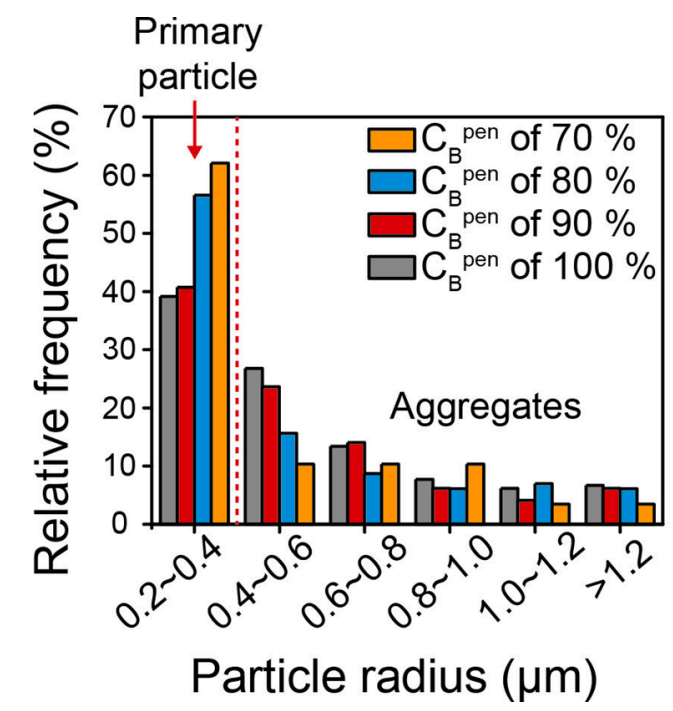

Fig. 6. Relative frequency of asphaltene particle size distribution with $C_{B}^{\text {pen }}$ of $70 \%$ to $100 \%$. which is consistent with the confocal result in Fig. 4. The proportion of the primary submicron particles increases with decrease of $C_{B}^{p e n}$, which means less aggregates are formed. In other word, the proportion of aggregates increases with increase of $C_{B}^{\text {pen }}$.

The final particles may represents the way how asphaltene precipitates evolve, triggered by mixing with n-pentane. Higher proportion of primary submicron particles at $C_{B}^{\text {pen }}$, as shown in Fig. 6 , is the consequence from an early stage of precipitation. More aggregates formed at higher $C_{B}^{\text {pen }}$ is equivalent to grown or aggregated precipitates. However, we do not know how the asphaltene aggregates from entities of even smaller sizes to primary submicron particles around $300 \mathrm{~nm}$ in radius, as the required spatial resolution is beyond the capacity of our technique.

\subsection{Effects of n-pentane concentration on precipitation dynamics}

We show the precipitates in situ during diffusive mixing process with a spatial resolution around $200 \mathrm{~nm}$ in this section. The spatial resolution of the measurement is compromised due to continuous change in the medium properties.

Fig. 7a show asphaltene precipitation precipitation process in situ. Time $0 s$ is defined as the appearance time of the first particle (radius $>$ $200 \mathrm{~nm}$ ) to be detected by TIRF. After a certain growth time, the surface coverage of the asphaltene particles in the observation area plateaus, as shown in Fig. 8. The asphaltene particle growth time (i.e. duration from first asphaltene particle to the surface coverage reaches plateau) are different for different groups of n-pentane concentration in solution B. As shown in Fig. 7b, the growth time at $C_{B}^{\text {pen }}$ of $70 \%$ and $80 \%$ are both around $20 \mathrm{~s}$, which is higher than the growth time at $C_{B}^{\text {pen }}$ of $90 \%$ and $100 \%$ around $5 s$.

After reaching plateau, the asphaltene primary submicron particle size was analyzed by the same method described in the Section 3.1. As shown in Fig. $7 \mathrm{c}$, the primary submicron particle size does not have detectable change after the growth time. Similar to the primary submicron particle radius at final state (i.e. after $5 \mathrm{~min}$ ), the primary submicron particle radius is around $300 \mathrm{~nm}$ for the four groups. There is also no obvious dependence of asphaltene primary submicron particle size on n-pentane composition in solution B.

Finally we note that the chemical composition of mixture in the main channel can be estimated using the brightness of TIRF images. In the time course images from TIRF measurements, the brightness is determined by the emission light intensity of the fluorophores in the evanescent field (Fig. S2 in Supporting information). Two factors affect the emission light intensity: fluorophore concentration and penetration depth. Within the excitation wavelength range used in our experiment, only dissolved asphaltene have appropriate fluorophores for emission. 
(a)
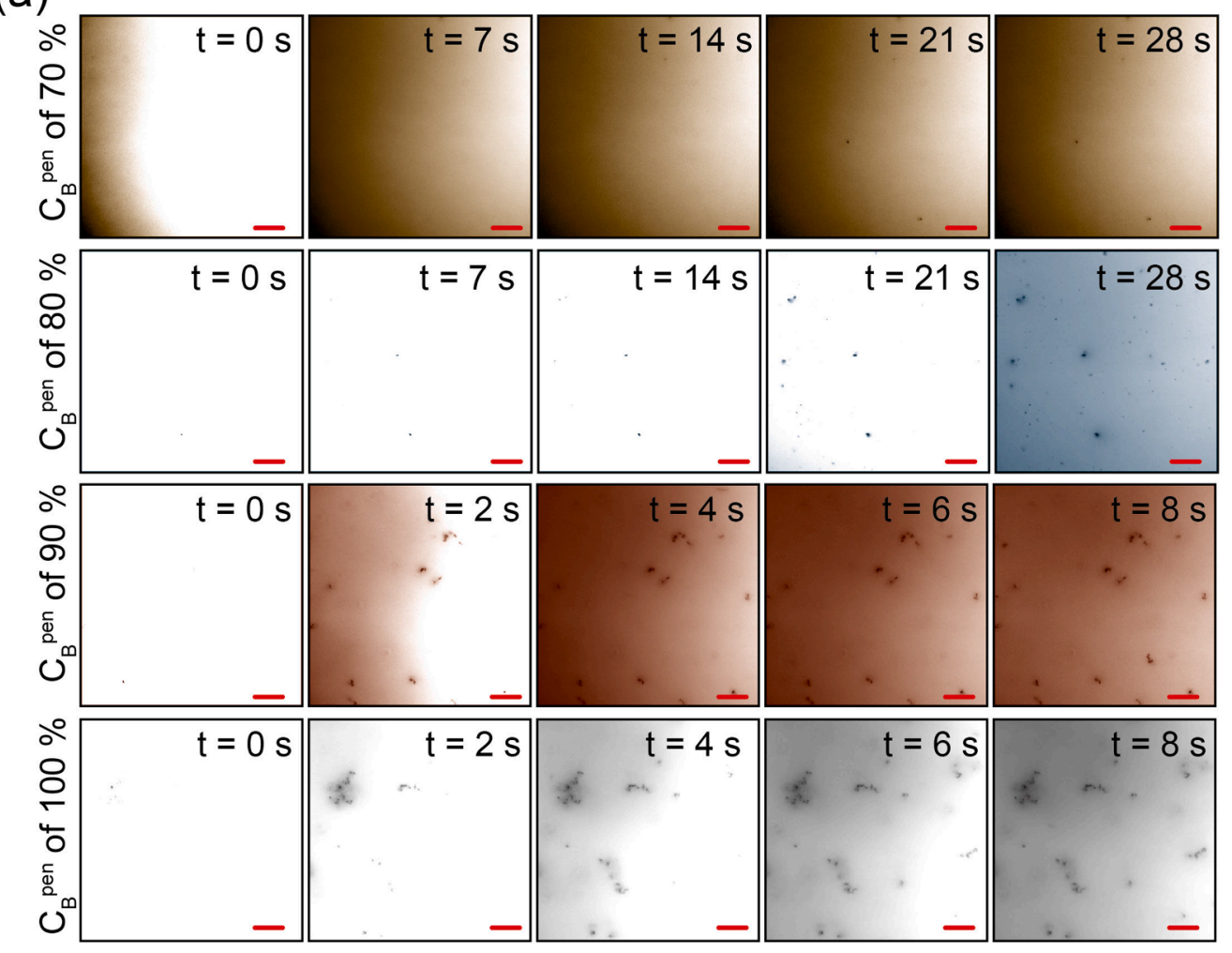

(b)

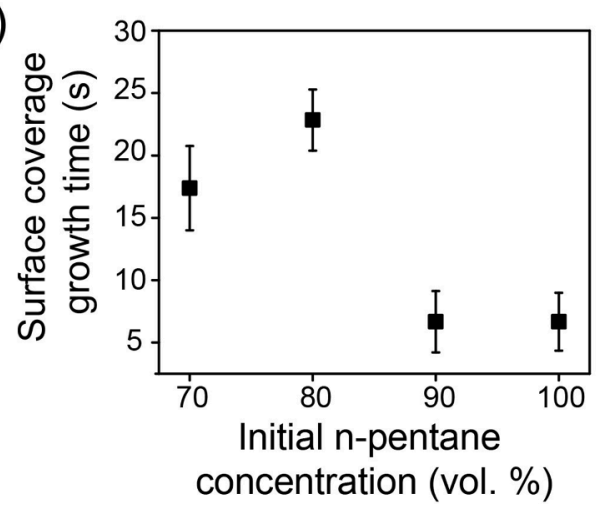

(c)

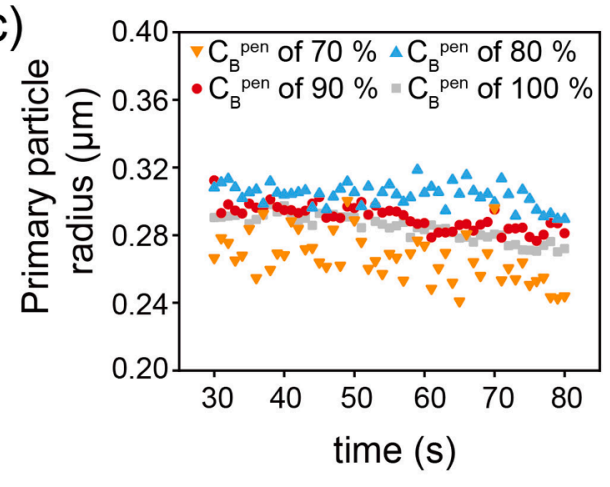

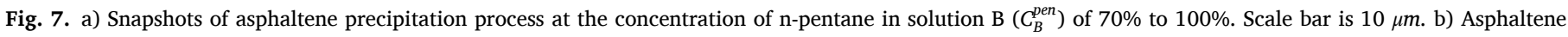

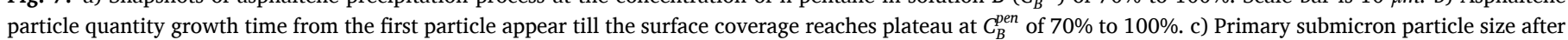
$30 s$.

Toluene and n-pentane have a weak emission (around 1000 a.u.), while precipitated asphaltene does not have obvious emission ( $<500$ a.u.). In the solvent mixing process, dissolved asphaltene concentration decreases, due to dilution by n-pentane. Asphaltene precipitation also decreases the dissolved asphaltene concentration, when $r_{\text {solvent/toluene }}$ is higher than the onset ratio. The refractive index $(R I)$ of n-pentane is 1.36 , lower than that of toluene $(R I=1.50)$. The decrease of penetration depth $\left(d_{p}\right)$ results in the decrease of excitation light intensity at a critical position during mixing. There is always a gradient from white to gray region from the mixing front. The signal from brightness can be obtained by gray scale value to estimate the solvent ratio. White region represents solution $\mathrm{A}$, while gray region represents solution $\mathrm{B}$ (i.e. n-pentane and toluene mixture). We tracked the shift in the position along x-direction with the same brightness intensity (i.e. same concentration of dissolved asphaltene) with time. The data shown in Supporting Information indeed suggest that the mixing process is dominated by diffusion.

\section{Discussion}

\subsection{Possible mechanism for presence of primary submicron particles}

The primary submicron particles observed by TIRF are not from settled precipitates under gravity as their sizes are too small to settle within the diffusion time of the solvent. Brownian motion of these particles is estimated as below [31]:

$\bar{X}_{\text {Brownian }}^{2}=\frac{4 R T K_{m} t}{3 \pi^{2} \eta N D}$

$\bar{X}_{\text {Brownian }}$ is the moving distance of particles due to Brownian motion. $R$ is gas constant. $T$ is temperature, which is $294 K . K_{m}$ is correction for discontinuity of fluid, which is 1 for liquid. $t$ is time of solvent diffuses across the observation field, which is 7.5 $s$ as shown in Fig. S2a and S2b. $\eta$ is the viscosity of the medium, which is assumed the same as the viscosity of n-pentane (i.e. $0.214 \mathrm{mPa} \cdot \mathrm{s}$ ) because n-pentane concentration is 
(a)

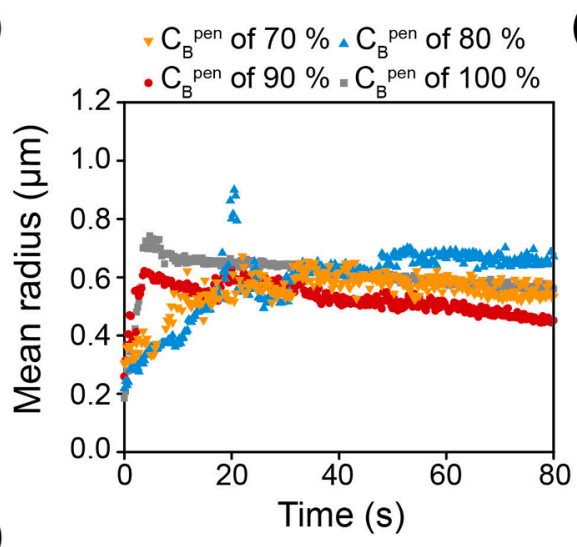

(c)

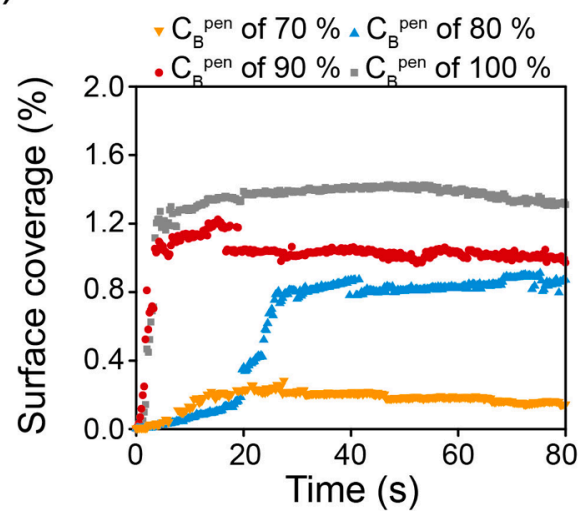

(b)

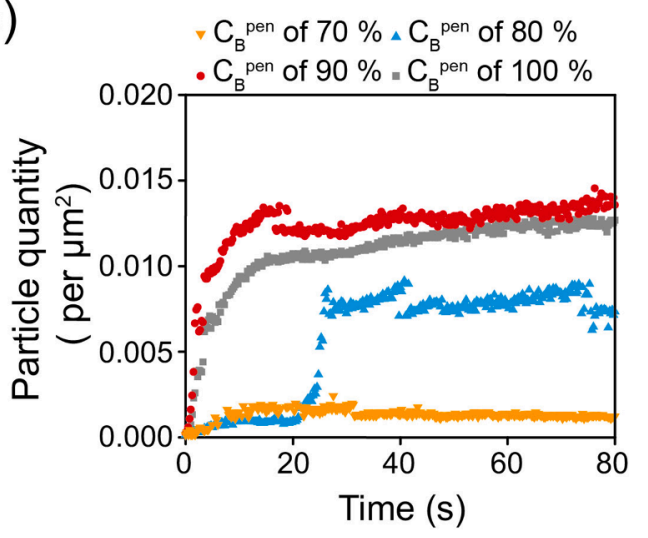

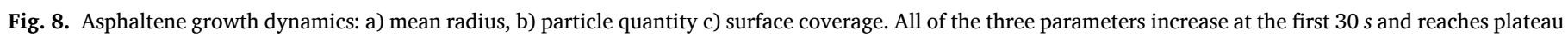
afterwards for the concentration of n-pentane in solution B $\left(C_{B}^{\text {pen }}\right)$ of $70 \%$ and $80 \%$. The plateau is much earlier for $C_{B}^{\text {pen }}$ of $90 \%$ and $100 \%$.

high when asphaltene precipitation happens. $N$ is Avogadro constant. $D$ is the diameter of particles. Stokes settling motion of particles is estimated as below [31]:

$\bar{X}_{\text {settling }}=\frac{\left(\rho_{a s p}-\rho_{\text {sol }}\right) g D^{2} t}{18 \eta}$

$\bar{X}_{\text {settling }}$ is moving distance of particles due to settling under gravity. $g$ is gravity constant. $\rho_{\text {asp }}$ is density of asphaltene, which is around 1176 $\mathrm{kg} / \mathrm{m}^{3}$ [32]. $\rho_{\text {sol }}$ is the density of the solution, which is assumed the same as the density of n-pentane (i.e. $626 \mathrm{~kg} / \mathrm{m}^{3}$ ). Solving Eq. (1) and (2), the calculation shows that asphaltene particles smaller than $0.7 \mu \mathrm{m}$ in diameter do not settle due to Brownian motion. Primary submicron particles are much smaller than $0.7 \mu \mathrm{m}$, therefore not mainly from settling but possibly from proportional to the particle concentration.

There are theoretical models in literature to describe asphaltene precipitation based either on phase separation or on colloid interaction [33-38,21]. To rationalize our experimental results, here we focus on the paradigm of the colloid theory for simplicity. The temporal evolution and the effects from asphaltene concentration observed in our results can be equivalently rationalized by a phase separation model.

According to the colloid theory of asphaltene precipitation [39-42], asphaltene dispersed in a solvent exist in the form of colloids (namely asphaltene nano-aggregates [43]) with the size a few nanometers far below the spatial resolution of our measurements [44]. Mixing asphaltene in toluene with a paraffinic solvent reduces solvent strength in the liquid medium, which may shift the dominant colloidal interactions between asphaltene nano-aggregates from steric repulsion to van der Waals attraction according to classic DLVO theory [37,45-47]. The concentration ratio of the paraffinic solvent to toluene $\left(r_{\text {solvent/toluene }}\right)$ in the mixture has to be above a critical value (i.e. onset) [46] to initiate asphaltene precipitation $[45,40]$. Those heavy aromatic species of asphaltene with the lowest solubility in the paraffinic solvent precipitate first from the mixture, some of which deposit on the surface and are captured as primary submicron particles in our experiments. Those primary submicron particles that still suspended in the mixture may grow by acting as preferential sites [37] for further aggregation and precipitation of lighter species of asphaltene.

Compared to the diffusion of the nano-aggregates, slow or fast precipitation of primary submicron particles would lead to different paths in increasing the sizes of the precipitates. In the case that precipitation kinetics is slower than the time for nano-aggregates to diffuse to the formed primary submicron particles, larger aggregates may form from growth by adding nano-aggregates, as well as from merging of more than one primary submicron particle. In the case that precipitation kinetics is faster than the diffusion of nano-aggregates to existing primary submicron particles, the precipitates may be mainly in the form of primary submicron particles. We believe that when the concentration of pentane is low (such as $C_{B}^{\text {pen }}$ of $70 \%$ or $80 \%$ ), our experiments more resemble the latter case that the precipitation of primary submicron particles is faster than the growth of primary submicron particles from nano-aggregates by diffusion. At a lower concentration of pentane in solution $\mathrm{B}$, asphaltene is more diluted before the threshold for precipitation is reached. With a small population of asphaltene in the mixture, the nano-aggregates take a longer time to diffuse into the formed primary submicron particles.

An interesting question is why primary submicron particles are observed in all $C_{B}^{\text {pen }}$. During diffusive mixing processes in our experiments, remaining soluble asphaltene in the mixture $\left(C_{\text {total }}^{\text {Asp }}\right)$ decreases with time at the rate determined by diffusion process of solution $B$ in the main channel. Hence how fast $C_{\text {total }}^{\text {Asp }}$ decreases is independent of the initial concentration of n-pentane in solution $\mathrm{B}\left(C_{B}^{\text {pen }}\right)$. However, $r_{\text {solvent/toluene }}$ in the mixture is consistently lower for solution B with lower $C_{B}^{p e n}$. What in common for all groups of solution B is the precipitation 


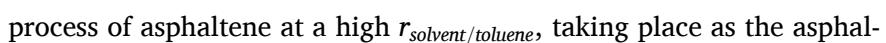
tene is nearly all depleted. Even for $C_{B}^{p e n}$ of $90 \%$ or $100 \%$, asphaltene is gradually depleted by the precipitation of primary submicron particles with time, the nano-aggregates become less likely to diffuse to the existing primary submicron particles. During this final process of precipitation, primary submicron particles still form due to the low solvation strength in the mixture. However, as the mixture is already sufficiently diluted by n-pentane, the number density of the primary submicron particles is low so that the probability for primary submicron particle collision and aggregates is also low. Therefore, primary submicron particles could be observed for all conditions. As even more paraffinic solvent is added to the mixture, $C_{\text {total }}^{\text {Asp }}$ eventually decreases to a certain limit and the precipitation terminates due to lack of sufficient asphaltene in the bulk solution. There are a low number of several large aggregates that may form from aggregates of discrete primary submicron particles, as shown in our images in Fig. 3 and 4.

Asphaltene precipitation at high dilution is consistent with the size distribution observed in our experiments for solution B with low $C_{B}^{\text {pen }}$. The precipitates mostly consist of primary submicron particles with fewer large aggregates (shown in Fig. 6) at $C_{B}^{\text {pen }}$ of $80 \%$ and $70 \%$. As comparison, the precipitated larger aggregates at $C_{B}^{\text {pen }}$ of $100 \%$ and $90 \%$ observed in our TIRF images may consist of many primary submicron particles or aggregates from primary submicron particles with nanoaggregates.

\subsection{Microscopic view of asphaltene precipitation dynamics}

We observed in Fig. 7 that the growth time at higher concentration of $\mathrm{n}$-pentane is shorter than that at lower concentration. Below we will rationalize this interesting result. To simplify the analysis of the asphaltene growth process, we made the following assumptions: (1) the first asphaltene particle is detected at the time that $r_{\text {solvent/toluene }}$ reaches onset, which is 1.22 (i.e. n-pentane concentration is $55 \mathrm{vol} . \%$ in the mixture) according to the value in the literature $[34,48]$. The exact number of onset is not a universal value. However, the following analysis holds for any number below $70 \%$. (2) The concentration ratio of asphaltene and toluene remains constant, determined by initial concentration of asphaltene in solution A. (a)

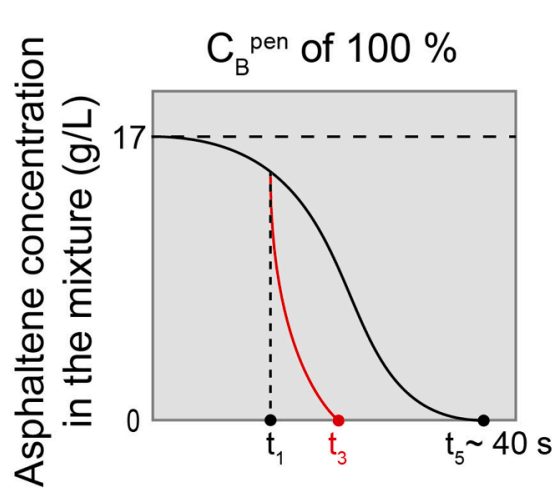

(c)

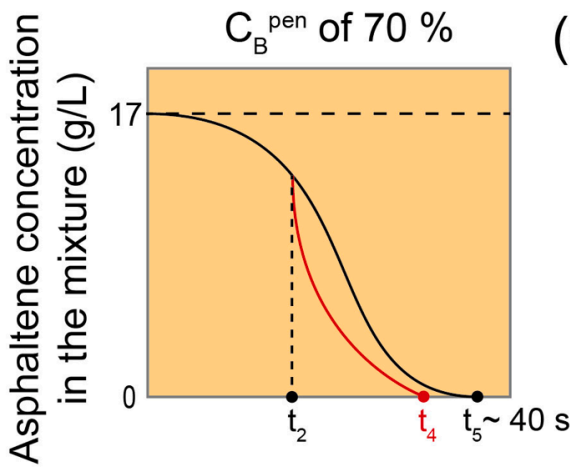

(b)

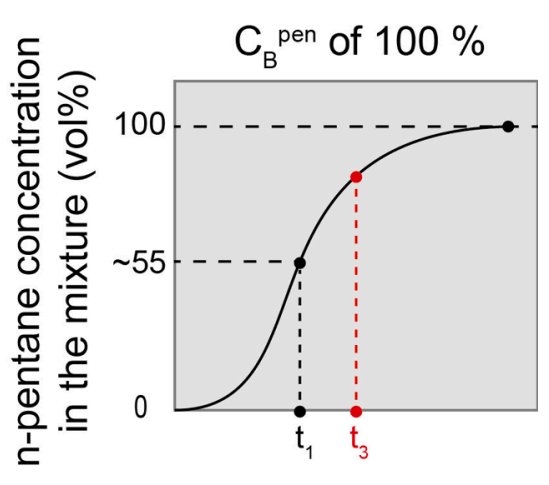

(d)

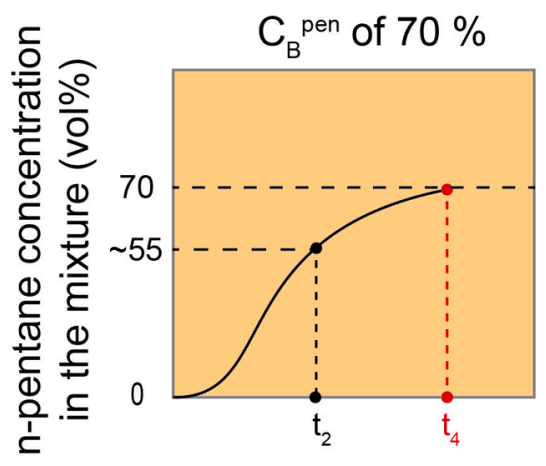

(e) $C_{B}{ }^{\text {pen }}$ of $100 \%$

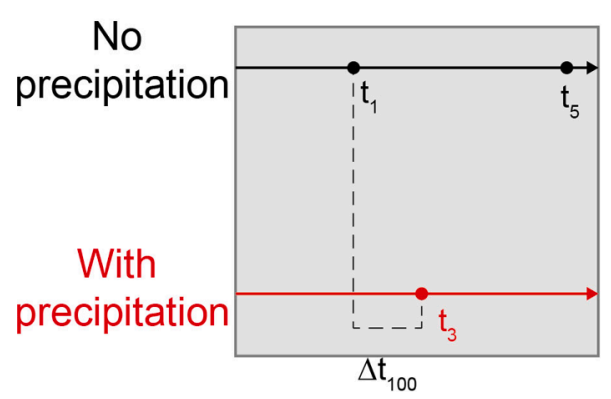

(f) $\mathrm{C}_{\mathrm{B}}^{\text {pen }}$ of $70 \%$

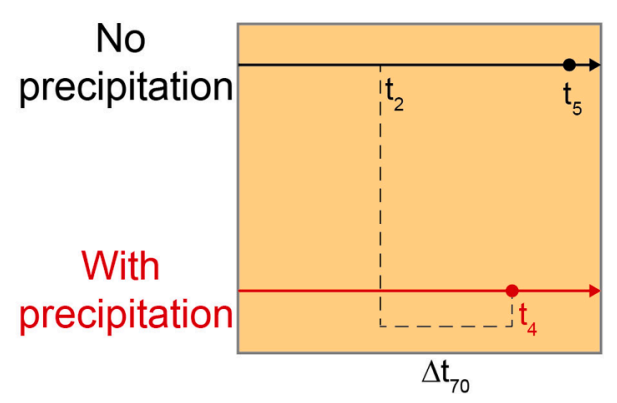

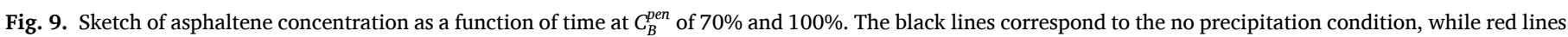

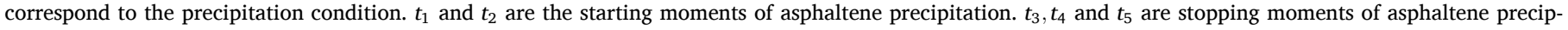
itation. The duration between the starting and stopping moments ( $\Delta t_{100}$ and $\Delta t_{70}$ are the growth time of asphaltene particles.). 
Based on the assumption, there are two important moments for asphaltene precipitation during diffusive mixing processes in the experiments: The time when $r_{\text {solvent/toluene }}$ reaches onset and the time when $C_{\text {total }}^{A s p}$ is too low and the precipitation terminates. As sketched in Fig. 9, the two moments are labelled as $t_{1}$ and $t_{3}$ for $C_{B}^{p e n}$ of $100 \%$, and $t_{2}$ and $t_{4}$ for $C_{B}^{p e n}$ of $70 \%$. The growth time $(\Delta t)$ is the time interval between these two moments, which is $\Delta t_{100}$ and $\Delta t_{70}$, respectively. $C_{\text {total }}^{A s p}$ in the mixture decreases from addition of solution $\mathrm{B}$, but also from the mass loss after the onset due to asphaltene precipitation. The growth time of asphaltene precipitation is governed by the relative kinetics of precipitation versus diffusion.

The growth time $\Delta t$ is estimated as below:

$\Delta t \sim \frac{c_{\text {onset }}}{k_{d} \dot{\omega}_{d}+k_{p} \dot{\omega}_{p}}$

Here $c_{\text {onset }}$ is the asphaltene concentration in the mixture when npentane concentration reaches onset. $\dot{\omega}_{d}$ is the reduction rate in $C_{\text {total }}^{\text {Asp }}$ in the mixture induced by dilution (black curve in the sketch), approximately the same for $C_{B}^{\text {pen }}$ of $100 \%$ and $70 \%$ as both are controlled by the diffusion process. $\dot{\omega}_{p}$ is the reduction rate in $C_{\text {total }}^{\text {Asp }}$ induced by precipitation (red curve in the sketch). $k_{d}$ and $k_{p}$ are coefficients of dilution and precipitation rate, respectively. $k_{d}$ and $k_{p}$ are different because dilution reduces all types of asphaltene but precipitation reduces the least soluble asphaltene. After the threshold concentration of n-pentane, the reduction of asphaltene concentration by precipitation can be much faster than by dilution at least for a short time window.

Based on colloidal theory, $\dot{\omega}_{p}$ can be estimated as below $[49,38,21]$ :

$\dot{\omega}_{p}=\alpha_{i, j} \beta_{i, j} c_{i} c_{j}$

Here $c_{i}$ and $c_{j}$ represent concentration of asphaltene particle with size $i$ and $j . \alpha_{i, j}$ and $\beta_{i, j}$ represent collision frequency and collision efficiency of asphaltene particle with size $i$ and $j$, respectively. The collision efficiency $\beta_{i, j}$ increases exponentially with n-alkane concentration [38]. Especially when $C_{B}^{\text {pen }}$ is higher than $80 \mathrm{vol}$.\% because the dominated force changes from repulsion to attraction (i.e. the energy barrier is much lower) [46]. Therefore $\dot{\omega}_{p}$ is larger. Accordingly, the growth time $\Delta t$ is shorter at larger $C_{B}^{\text {pen }}$ based on Eq. (3). In other words, with higher $C_{B}^{\text {pen }}$, the drop of $C_{\text {total }}^{A s p}$ is sharper, due to faster precipitation. The asphaltene is depleted in a shorter period of time and the time window for precipitation is narrower. In contrast, with lower $C_{B}^{\text {pen }}$, the precipitation rate of asphaltene is slow, so asphaltene almost grows until the end of solvent displacement.

In our experiments, $r_{\text {solvent/toluene }}$ at any given time could be estimated from the composition measurements using fluorescence intensities from diffusive mixing between toluene doped with fluorescent dye and pure n-pentane. Assuming that fluorescent dye and toluene ratio remains constant, we can estimate compositions of toluene and n-pentane in the mixture in the main channel of the microchamber. Details of the mixing characterization are reported elsewhere. [25] Assuming that asphaltene precipitation after $r_{\text {solvent/toluene }}$ of 4 (i.e. n-pentane concentration is 80 vol.\% in the mixture) does not contribute much to the aggregates observed at high $C_{B}^{p e n}$, we estimated that it was approximately $5.5 s$ and $8.5 s$ at $C_{B}^{\text {pen }}$ of $90 \%$ and $100 \%$, respectively, to reach $r_{\text {solvent/toluene }}$ of 4 from the onset of 1.22 . The duration is consistent with the growth time $\Delta t$ of $90 \%$ and $100 \%$. Our measurements were not sensitive enough to distinguish if there is systematic difference between these two, but is enough to identify the difference between two high and two low groups of $C_{B}^{\text {pen }}$.

\subsection{Implications to asphaltene colloid theory and paraffinic froth treatment}

A question is why the primary submicron particles observed under various conditions are similar in size, all around $200-400 \mathrm{~nm}$ in radius.
These particles were not observed in toluene solution at the same spatial resolution of our images. Clearly, the formation of primary submicron particles was induced by addition of n-pentane, after the solvation strength in the liquid medium was reduced. As solvation strength reduces by addition of n-pentane, asphaltene nano-aggregates may reorganize and form more compact and larger entities that become visible to our imaging technique, due to higher density relative to the surrounding liquid medium. The compact asphaltene entities stranded in the field of view were captured and monitored by our images. The reorganized and compact asphaltene entities formed at a low concentration of asphaltene at high dilution by n-pentane may be the primary submicron particles observed in our experiments. Similar sizes of primary submicron particles formed at low concentration of asphaltene (near the end of solvent diffusion even for $C_{B}^{\text {pen }}$ of $100 \%$ ) could be due to that the formation process of the particles up to this size of $300 \mathrm{~nm}$ is fast, relative to dilution of asphaltene by diffusive mixing accompanied by the change in the solvation effect.

As mentioned in the introduction, paraffinic froth treatment (PFT) is an essential step in oilsands extraction to remove solids and water from bitumen froth through dilution by paraffinic solvents. Although PFT and many other solvent-induced phase separation processes are conducted through convective mixing, clearly local diffusive mixing is unavoidable, even for the case in a turbulence flow. In particular, for a large volume of liquid, viscous liquid or mixing confined in a small space, diffusive mixing may play a role as it is challenging to reach uniform mixing instantly. Before being uniformly mixed, the added solvent diffuses into bitumen. The early stage phase separation of asphaltene influences the number density, size distribution and morphology of the precipitates, which may impact the subsequent agglomeration with fine solids and water droplets and their settlement in PFT process. Our results showed that when n-pentane concentration is high and asphaltene concentration is low, the precipitates are mainly in the form of primary submicron particles that may not contribute to the settling of water and solids directly, due to their small surface area, and slow settling rate under gravity. Moreover, it was also believed that water droplets in PFT are coated with asphaltene due to their surface activity [2]. The addition of a poor solvent may cause the formation of small asphaltene particles at droplet surface. The size of the asphaltene particles on water drops may play an important role in the settling process, because the interfacial properties of water drops may depend on the size of asphaltene particles. In analogy, the nanoparticle size and shape are essential to the stability of Pickering emulsions [50,51]. As compared to larger particles, it is likely that small asphaltene particle may stabilize water droplets by reducing their coalescence, and hence hinder the separation process in PFT.

\section{Conclusions}

In this work, the asphaltene precipitation under diffusive mixing is tracked in situ by TIRF microscope in a quasi-2D chamber. Asphaltene precipitation occurs simultaneously during diffusion of $n$-pentane into the asphaltene solution. As n-pentane to toluene ratio exceeds onset with mixing, the interaction between asphaltene colloids changes from repulsion to attraction to form a new phase as precipitation. The asphaltene precipitation stops when the asphaltene concentration is not enough to provide source of asphaltene for precipitation. The diffusive mixing lasts for a few seconds and the asphaltene precipitation lasts for a similar time scale $(<30 s)$. Two types of asphaltene particles (i.e. primary submicron particles and aggregates) are formed in the dilution induced precipitation. More aggregates are formed with higher initial npentane concentration, but the primary submicron particles with a similar size range from $200 \mathrm{~nm}$ to $400 \mathrm{~nm}$ in radius are observed independent of n-pentane concentration. The findings from this project will provide new insight into the effects of solvent mixing on the onset, size distribution and morphological characteristics of asphaltene 
precipitates. This fundamental understanding may help design mixing conditions to achieve enhanced separation.

In addition to the parameters studied in this study, TIRF provides an opportunity for future work to investigate the effects of many other interesting parameters that are important to asphaltene precipitation. Some examples are type and initial concentration of asphaltene, temperature, precipitant type, solid contents and others.

\section{CRediT authorship contribution statement}

Jia Meng: Methodology, Investigation, Formal analysis, Writing original draft. Jae Bem You: Writing - review \& editing. Hao Hao: Software. Xiaoli Tan: Resources, Supervision, Writing - review \& editing. Xuehua Zhang: Conceptualization, Writing - review \& editing, Supervision.

\section{Declaration of Competing Interest}

The authors declare that they have no known competing financial interests or personal relationships that could have appeared to influence the work reported in this paper.

\section{Acknowledgements}

The authors acknowledge the funding support from the Institute for Oil Sands Innovation (IOSI) (project number IOSI 2018-03) and from the Natural Science and Engineering Research Council of Canada (NSERC)-Collaborative Research and Development Grants. The authors are grateful for technical support from IOSI lab, particularly from Lisa Brandt and Brittany MacKinnon. We are also grateful for the technical support of Dr. Xuejun Sun at the Cell Imaging Facility at the Cross Cancer Institute and Dr. Murray R. Gray in Alberta Innovates for fruitful discussions. We are grateful for the valuable comments from anonymous reviewers.

\section{Appendix A. Supplementary data}

Supplementary data associated with this article can be found, in the online version, athttps://doi.org/10.1016/j.fuel.2021.120584.

\section{References}

[1] Taylor SD, Czarnecki J, Masliyah J. Refractive index measurements of diluted bitumen solutions. Fuel 2001;80:2013-8.

[2] Rao F. Liu, Q, Froth Treatment in Athabasca Oil Sands Bitumen Recovery Process: A Review. Energy Fuels 2013;27:7199-207.

[3] Xu Y. Asphaltene Precipitation in Paraffinic Froth Treatment: Effects of Solvent and Temperature. Energy Fuels 2018;32:2801-10.

[4] Casas YA, Duran JA, Schoeggl FF, Yarranton HW. Settling of Asphaltene Aggregates in n-Alkane Diluted Bitumen. Energy Fuels 2019;33:10687-703.

[5] Yudin I, Nikolaenko G, Gorodetskii E, Kosov V, Melikyan V, Markhashov E, Frot D, Briolant Y. Mechanisms of asphaltene aggregation in toluene-heptane mixtures. J Petrol Sci Eng 1998;20:297-301.

[6] Li X, Guo Y, Boek ES, Guo X. Experimental Study on Kinetics of Asphaltene Aggregation in a Microcapillary. Energy Fuels 2017;31:9006-15.

[7] Seifried CM, Crawshaw J, Boek ES. Kinetics of Asphaltene Aggregation in Crude Oi Studied by Confocal Laser-Scanning Microscopy. Energy Fuels 2013;27:1865-72.

[8] Zhang X, Lu Z, Tan H, Bao L, He Y, Sun C, Lohse D. Formation of surface nanodroplets under controlled flow conditions. Proc National Acad Sci USA 2015; 112:9253-7.

[9] Vitale S, Katz J. Liquid droplet dispersions formed by homogeneous liquid/liquid nucleation: "The ouzo effect". Langmuir 2003;19:4105-10.

[10] Zemb TN, Klossek M, Lopian T, Marcus J, Schöettl S, Horinek D, et al. How to explain microemulsions formed by solvent mixtures without conventional surfactants. Proc National Acad Sci USA 2016;113:4260-5.

[11] Rezaee M, Assadi Y, Hosseini M-RM, Aghaee E, Ahmadi F, Berijani S. Determination of organic compounds in water using dispersive liquid-liquid microextraction. J Chromatogr A 2006;1116:1-9.

[12] Zhang XH, Ren J, Yang H, He Y, Tan J, Qiao GG. From transient nanodroplets to permanent nanolenses. Soft Matter 2012;8:4314-7.

[13] Schubert S, DelaneyJr JT, Schubert US. Nanoprecipitation and nanoformulation of polymers: from history to powerful possibilities beyond poly(lactic acid). Soft Matter 2011;7:1581-8.
[14] Aubry J, Ganachaud F, CohenAddad J-P, Cabane B. Nanoprecipitation of Polymethylmethacrylate by Solvent Shifting:1. Boundaries. Langmuir 2009;25: 1970-9.

[15] Lepeltier E, Bourgaux C, Couvreur P. Nanoprecipitation and the "ouzo effect": Application to drug delivery devices. Adv Drug Deliv Rev 2014:71:86-97.

[16] Lu Z, Xu H, Zeng H, Zhang X. Solvent effects on the formation of surface nanodroplets by solvent exchange. Langmuir 2015;31:12120-5.

[17] Castillo J, Hung J, Goncalves S, Reyes A. Study of Asphaltenes Aggregation Process in Crude Oils Using Confocal Microscopy. Energy Fuels 2004;18:698-703.

[18] Hung J, Castillo J, Reyes A. Kinetics of Asphaltene Aggregation in Toluene? Heptane Mixtures Studied By Confocal Microscopy. Energy Fuels 2005;19: 898-904.

[19] Axelrod, D. In Fluorescence Microscopy of Living Cells in Culture Part B. Quantitative Fluorescence Microscopy-Imaging and Spectroscopy; Taylor, D.L., Wang, Y.-L., Eds., Methods in Cell Biology; Academic Press, 1989; Vol. 30; pp 245-270.

[20] Duran J, Casas Y, Xiang L, Zhang L, Zeng H, Yarranton H. Nature of asphaltene aggregates. Energy Fuels 2018;33:3694-710.

[21] Duran J, Schoeggl F, Yarranton H. Kinetics of asphaltene precipitation/aggregation from diluted crude oil. Fuel 2019;255:115859.

[22] Long Y, Dabros T, Hamza H. Structure of water/solids/asphaltenes aggregates and effect of mixing temperature on settling rate in solvent-diluted bitumen. Fuel 2004; 83:823-32.

[23] Long Y, Dabros T, Hamza H. Stability and settling characteristics of solvent-diluted bitumen emulsions. Fuel 2002;81:1945-52.

[24] Lu Z, Schaarsberg MHK, Zhu X, Yeo LY, Lohse D, Zhang X. Universal nanodroplet branches from confining the Ouzo effect. Proc National Acad Sci USA 2017, 114, 10332-10337.

[25] Meng J, You JB, Arends GF, Hao H, Tan X, Zhang X. Microfluidic device coupled with total internal reflection microscopy for in situ observation of precipitation. arXiv e-prints 2020, arXiv:2012.06962.

[26] Buckley J, Hirasaki G, Liu Y, VonDrasek S, Wang J, Gill B. Asphaltene precipitation and solvent properties of crude oils. Pet Sci Technol 1998;16:251-85.

[27] Wattana P, Wojciechowski DJ, Bolaos G, Fogler H. Study of asphaltene precipitation using refractive index measurement. Pet Sci Technol 2003, 21, 591-613.

[28] Kedenburg S, Vieweg M, Gissibl T, Giessen H. Linear refractive index and absorption measurements of nonlinear optical liquids in the visible and nearinfrared spectral region. Opt Mater Express 2012;2:1588-611.

[29] Paddock SW. Confocal laser scanning microscopy. Biotechniques 1999;27: 992-1004.

[30] Sirota E. Swelling of asphaltenes. Pet Sci Technol 1998;16:415-31.

[31] Allen T. Particle size measurement. Springer; 1990.

[32] Barrera D, Ortiz D, Yarranton H. Molecular weight and density distributions of asphaltenes from crude oils. Energy Fuels 2013;27:2474-87.

[33] Rahimi H, SolaimanyNazar AR. Asphaltene aggregates fractal restructuring model, a population balance approach. Energy Fuels 2010;24:1088-93.

[34] Agrawal P, Schoeggl F, Satyro M, Taylor S, Yarranton H. Measurement and modeling of the phase behavior of solvent diluted bitumens. Fluid Phase Equilib 2012;334:51-64.

[35] Torkaman M, Bahrami M, Dehghani M. Influence of Temperature on Aggregation and Stability of Asphaltenes. I. Perikinetic Aggregation. Energy Fuels 2017;31; 11169-80.

[36] Subramanian S, Simon S, Sjöblom J. Asphaltene precipitation models: a review. J Dispersion Sci Technol 2016;37:1027-49.

[37] Gray MR, Tykwinski RR, Stryker JM, Tan X. Supramolecular assembly model for aggregation of petroleum asphaltenes. Energy Fuels 2011;25:3125-34.

[38] Maqbool T, Raha S, Hoepfner MP, Fogler HS. Modeling the aggregation of asphaltene nanoaggregates in crude oil- precipitant systems. Energy Fuels 2011;25: 1585-96.

[39] Zhao B, Shaw JM. Composition and size distribution of coherent nanostructures in Athabasca bitumen and Maya crude oil. Energy Fuels 2007;21:2795-804.

[40] Ollinger J, Pourmohammadbagher A, Quast AD, Becerra M, Shumaker-Parry JS, Shaw JM. Gold Core Nanoparticle Mimics for Asphaltene Behaviors in Solution and at Interfaces. Energy Fuels 2016;30:10148-60.

[41] Gray MR, Yarranton HW. Quantitative Modeling of Formation of Asphaltene Nanoaggregates. Energy Fuels 2019;33:8566-75.

[42] Derakhshesh M, Gray MR, Dechaine GP. Dispersion of asphaltene nanoaggregates and the role of Rayleigh scattering in the absorption of visible electromagnetic radiation by these nanoaggregates. Energy Fuels 2013;27:680-93.

[43] Mullins OC, Sabbah H, Eyssautier J, Pomerantz AE, Barré L, Andrews AB, RuizMorales Y, Mostowfi F, McFarlane R, Goual L, et al. Advances in asphaltene science and the Yen-Mullins model. Energy Fuels 2012;26:3986-4003.

[44] Yarranton HW, Ortiz D, Barrera D, Baydak E, Barré L, Frot D, Eyssautier J, Zeng H, Xu Z, Dechaine G, et al. On the size distribution of self-associated asphaltenes. Energy Fuels 2013;27:5083-106.

[45] Anisimov M, Ganeeva YM, Gorodetskii E, Deshabo V, Kosov V, Kuryakov V, Yudin D, Yudin I. Effects of resins on aggregation and stability of asphaltenes. Energy Fuels 2014;28:6200-9.

[46] Wang S, Liu J, Zhang L, Masliyah J, Xu Z. Interaction forces between asphaltene surfaces in organic solvents. Langmuir 2010;26:183-90.

[47] Wang S, Liu J, Zhang L, Xu Z, Masliyah J. Colloidal interactions between asphaltene surfaces in toluene. Energy Fuels 2009;23:862-9.

[48] Johnston K, Schoeggl F, Satyro M, Taylor S, Yarranton H. Phase behavior of bitumen and n-pentane. Fluid Phase Equilib 2017;442:1-19. 
[49] Thomas D, Judd S, Fawcett N. Flocculation modelling: a review. Water Res 1999; 33:1579-92.

[50] Bizmark N, Ioannidis MA. Nanoparticle-stabilised emulsions: droplet armouring vs. droplet bridging. Soft Matter 2018;14:6404-8.
[51] Weijgertze HM, Kegel WK, Zanini M. Patchy rough colloids as Pickering stabilizers. Soft Matter 2020;16:8002-12. 